This can be proved by combining the two formulas in Elliott's paper in the Messenger of Mathematics, volume 7 (1878), page 151 and in Minkowski's paper in the Mathematische Annalen, volume 57 (1903), page 463.

But any similar formula for the relative volume of two convex ovoid bodies cannot be established.

March, 1918.

\title{
CONCERNING THE DEFINITION OF A SIMPLE CONTINUOUS ARC.
}

BY DR. GEORGE H. HALLETT, JR,

(Read before the American Mathematical Society October 26, 1918.)

IN a paper entitled "Curves in non-metrical analysis situs with an application in the calculus of variations," American Journal of Mathematics, volume 33 (1911), pages 285-326, N. J. Lennes gives the following definition of a simple continuous arc.*

"A continuous simple arc connecting two points $A$ and $B$, $A \neq B$, is a bounded, closed, connected set of points $[A]$ containing $A$ and $B$ such that no connected proper subset of $[A]$ contains $A$ and $B . "$

I shall show that the word "bounded" in this definition is superfluous. $\dagger$

Lennes proves the simpler properties of formal order on an arc without any use of the assumption that it is bounded. He also proves $(\$ \S 4,8)$ that "if $A_{0}$ is any point of an arc $A B$, and $t_{1}$ any triangle containing $A_{0}$ as an interior point, then (in case $A_{0} \neq A$ ) there is a point $A_{1}$ on the arc $A A_{0}$ and (in case $A_{0} \neq B$ ) a similar point $\mathrm{B}_{1}$ on the arc $B A_{0}$ such that every point of the $\operatorname{arc} A_{1} B_{1}$ lies within $t_{1}$."

The following theorem also follows readily without use of the assumption that an arc is bounded:

If a point $A_{0}$ of an arc $A B$ is a limit point of a set of points [S] of the $\operatorname{arc} A B$, and $C$ is $A$ or (if $A_{0} \neq A$ ) any point of the

* Loc. cit., p. 308.

$\dagger$ Since I wrote this paper it has been pointed out to me by Professor R. L. Moore that a modification of the argument used in the proof of Theorem 49 on p. 159 of his paper "On the foundations of plane analysis situs," Transactions Amer. Math. Society, vol. 17 (1916), pp. 131-164, would accomplish the same result. 
subarc $A A_{0}$ of $A B$, and $D$ is $B$ or (if $A_{0} \neq B$ ) any point of the subarc $A_{0} B$ of $A B$, then the subarc $C D$ of $A B$ contains at least one point of $[S]$.

This theorem is tacitly assumed by Lennes in his proof of Theorem 7 (\$4).

All the above mentioned theorems thus hold if the term simple continuous arc is defined without the use of the word "bounded." Using this definition of an arc, I now prove the

Theorem. A simple continuous arc is bounded.

Proof. Suppose $A B$ is an arc which is not bounded. Let [ $\left.S_{1}\right]$ consist of $A$ and all points $S_{1}$ of $A B$ such that the arc $A S_{1}$ is bounded. Let $\left[S_{2}\right]$ consist of all other points of $A B$. By hypothesis both $\left[S_{1}\right]$ and $\left[S_{2}\right]$ exist. No point of $S_{2}$ is between $A$ and a point of $\left[S_{1}\right]$. Since $A B$ is connected, $\left[S_{1}\right]$ contains a limit point $P_{1}$ of $\left[S_{2}\right]$ or $\left[S_{2}\right]$ contains a limit point $P_{2}$ of $\left[S_{1}\right]$. In the first case any triangle $t_{1}$ containing $P_{1}$ contains an arc $a_{1}$ of $A B$ containing $P_{1}$. The arc $a_{1}$ contains a point $Q_{2}$ of $\left[S_{2}\right]$. The $\operatorname{arc} A P_{1}$ of $A B$ is contained in a polygon $p_{1}$. Therefore the subarc $A Q_{2}=A P_{1}+P_{1} Q_{2}$ lies entirely within a polygon (Lennes, Theorem $15, \S 2$ ), and is bounded, contrary to hypothesis. In the second case any triangle $t_{2}$ about $P_{2}$ contains an arc $a_{2}$ containing $P_{2}, a_{2}$ contains a point $Q_{1}$ of $\left[S_{1}\right], A Q_{1}$ is contained in a polygon, and therefore $A P_{2}=A Q_{1}$ $+Q_{1} P_{2}$ is contained in a polygon and is bounded, contrary to hypothesis. Thus in either case the supposition that $A B$ is not bounded leads to a contradiction.

University of Pennsylvania.

\title{
THE TRANSFORMATION OF A REGULAR GROUP INTO ITS CONJOINT.
}

\author{
BY DR. J. E. MCATEE,
}

(Read before the American Mathematical Society October 26, 1918.)

1. Consider a regular substitution group $G$ of order $g$. All the substitutions on the same letters that are commutative with every substitution of $G$ form a group $G^{\prime}$, of order $g$, called the conjoint of $G$. These groups are conjugate.* If $G$ is abelian, $G=G^{\prime}$. In the contrary case the statement that a

* Finite Groups, Miller, Blichfeldt and Dickson, 1916, p. 35. 